\title{
Docosahexanoic acid modifies low-density lipoprotein receptor abundance in HepG2 cells via suppression of the LXR $\alpha$-Idol pathway
}

\author{
YING ZHOU, YUE GUO, XIAODONG ZHUANG and ZHIMIN DU \\ Department of Cardiology, Key Laboratory on Assisted Circulation, First Affiliated Hospital, \\ Sun Yat-sen University, Guangzhou, Guangdong 510080, P.R. China \\ Received February 16, 2014; Accepted October 24, 2014
}

DOI: $10.3892 / \mathrm{mmr} .2014 .2940$

\begin{abstract}
As a daily supplement, omega-3 fatty acid is confirmed to be of benefit in hypertriglyceridemia. However, the effect of omega-3 fatty acids on the low-density lipoprotein cholesterol (LDL-C) metabolism remains a controversial issue. In this study, we focused on the regulatory effect of docosahexanoic acid (DHA), one type of omega-3 fatty acid, exerted on the LDL receptor (LDLR), a determinant regulator of the LDL-C metabolism, and explored the potential mechanism. We observed that DHA increased hepatic LDLR protein in the presence of 25-hydroxycholesterol in HepG2 cells but did not alter the mRNA level. Previous studies have identified inducible degrader of the LDLR (Idol) as a novel negative post-translational modulator of LDLR and a direct transcriptional target of liver $\mathrm{X}$ receptor $\alpha(\mathrm{LXR} \alpha)$. Since DHA had no effect on the transcriptional level of LDLR, we speculated that the post-transcriptional pathway LXR $\alpha$-Idol participated in this regulation. The results reveal that DHA downregulated the expression of $\mathrm{LXR} \alpha$ and Idol in coordination with the upregulation of LDLR expression. Multiple mechanisms are involved in the regulation of LDLR by DHA, and the suppression of the LXR $\alpha$-Idol pathway is one of these mechanisms.
\end{abstract}

\section{Introduction}

Low-density lipoprotein cholesterol (LDL-C) has been identified to have a crucial and causal role in the genesis of coronary heart disease and atherosclerotic cardiovascular disease (ASCVD) (1). There is notable evidence to suggest that higher LDL-C levels are correlated with greater ASCVD risk, and

Correspondence to: Professor Zhimin Du, Department of Cardiology, Key Laboratory on Assisted Circulation, First Affiliated Hospital, Sun Yat-sen University, 58 Zhongshan Road II, Guangzhou, Guangdong 510080, P.R. China

E-mail: zsy_dzm@126.com

Key words: docosahexanoic acid, low-density lipoprotein receptor, inducible degrader of the low-density lipoprotein receptor, liver $\mathrm{X}$ receptor $\alpha$ that lowering cholesterol levels reduces ASCVD events (2-5). Hepatic LDL receptor (LDLR) is essential for the uptake of extracellular LDL-C (6). As such, it is a determinant regulator of the LDL-C metabolism. One of the most optimal strategies to lower LDL-C is to upregulate and stabilize hepatic LDLR expression.

The abundance of LDLR is noted on the transcriptional and post-transcriptional levels. On the transcriptional level, LDLR is tightly regulated by sterol response element binding protein 2 (SREBP2) $(7,8)$. Cellular cholesterol depletion activates the nuclear translocation of SREBP2, and subsequently SREBP2 activates LDLR and proprotein convertase subtilisin/ kexin type 9 (PCSK9) gene expression (7-9). PCSK9 plays a pivotal role in the post-transcriptional regulation of LDLR, in which it binds to the extracellular domain LDLR and directs the trafficking of it to the lysosomes for degradation (10). Previous studies have identified a new significant degrader of LDLR, named inducible degrader of the LDLR (Idol), which is an E3 ubiquitin ligase that triggers ubiquitination of LDLR on its cytoplasmic domain and promotes lysosomal degradation (11). Distinct from PCSK9, Idol is regulated by another sterol-dependent nuclear receptor, liver $\mathrm{X}$ receptor $\alpha$ $(\mathrm{LXR} \alpha)$, which is activated in response to cellular cholesterol excess (12).

Omega-3 fatty acids, including docosahexanoic acid (DHA, 22:6n-3) and eicosapentaenoic acid (EPA, 20:5n-3), are also known as marine fatty acids, and are delivered from dietary fish oil. In vivo and in vitro studies confirmed that DHA and EPA are potent inhibitors of $\operatorname{LXR} \alpha(13,14)$. In addition, several studies have identified that omega-3 fatty acids upregulate LDLR abundance $(15,16)$. However, little is known about the mechanism by which fatty acids regulate LDLR. We considered that the newly identified LXR $\alpha$ target gene Idol may participate in this process. In the present study, we selected DHA as one type of omega-3 fatty acid to search for the possible mechanism by which these fatty acids exert their regulatory effects on LDLR. The results revealed that DHA increased hepatic LDLR abundance through the suppression of Idol expression rather than through gene expression. Furthermore, this repression of LXR $\alpha$ activity by DHA and the subsequent inhibition of the expression of Idol is one of multiple mechanisms. 


\section{Materials and methods}

Reagents. Palmitic acids (PA), DHA and 25-hydroxycholesterol were purchased from Sigma-Aldrich (St. Louis, MO, USA). Fatty acid-free bovine serum albumin (BSA) was obtained from MP Biomedicals (Santa Ana, CA, USA). Cell culture reagents were purchased from Gibco (Carlsbad, CA, USA). BCA protein assay was purchased from Thermo Fisher Scientific (Waltham, MA, USA). PVDF membranes and the ECL western blot system were provided by Merck Millipore (Billerica, MA, USA). Anti-LXR alpha antibody (ab176323) and anti-Idol antibody (ab74562) were obtained from Abcam (Cambridge, MA, USA); anti-LDLR antibody (10007665) from Cayman Chemical Co. (Ann Arbor, MI, USA); and anti-glyceraldehyde 3-phosphate dehydrogenase (GAPDH) antibody, peroxidase-conjugated anti-mouse antibody and anti-rabbit antibody from Proteintech Group (Chicago, IL, USA). The Ultrapure RNA kit was purchased from CWBIO (Beijing, China). The RevertAid First Strand cDNA synthesis kit (K1622) was purchased from Thermo Fisher Scientific. iTaq ${ }^{\mathrm{TM}}$ Universal SYBR $^{\circledR}$ Green supermix (172-5121) was purchased from Bio-Rad (Hercules, CA, USA).

Cell cultures and treatments. The human hepatoma HepG2 cell line, obtained from Laboratory Animal Center (Sun Yat-sen University, Guangzhou, China), was cultured in low-glucose Dulbecco's modified Eagle's medium (DMEM) supplemented with $10 \%$ fetal bovine serum at $37^{\circ} \mathrm{C}$ in a humidified atmosphere of $5 \% \mathrm{CO}_{2}$. For experiments, cells were seeded at a density of $1 \times 10^{6}$ cells per well and allowed to adhere for $24 \mathrm{~h}$. Each fatty acid was dissolved in ethanol and mixed with fatty acid-free BSA at 2.5:1 molar ratios, working as a fatty acid/BSA complex. 25-hydroxycholesterol was dissolved in ethanol and used at a concentration of $10 \mu \mathrm{mol} / 1$. Following the replacement of serum-free medium at $24 \mathrm{~h}$, fatty acid/BSA complexes were added to the culture dishes at a concentration of $100 \mu \mathrm{mol} / 1$ fatty acid and $0.25 \%$ BSA, with or without 25-hydroxycholesterol. Control cells were treated with BSA vehicle with or without 25-hydroxycholesterol. For dose-response experiments, 25-hydroxycholesterol and increasing concentrations $(50,100$ and $200 \mu \mathrm{mol} / \mathrm{l})$ of DHA were added to the culture media. After $24 \mathrm{~h}$, cells were either harvested for protein extraction or RNA isolation.

Western blot analysis. Following the treatments, cells were washed with phosphate-buffered saline, lysed in cell lysis buffer and incubated at $4^{\circ} \mathrm{C}$ for $20 \mathrm{~min}$, then centrifuged at $10,000 \mathrm{xg}$ for $10 \mathrm{~min}$ at $4^{\circ} \mathrm{C}$. Protein concentration was measured using the BCA method. Twenty micrograms of total proteins from each extract were separated by $8 \%$ or $10 \%$ SDS-polyacrylamide gels and transferred onto PVDF membranes in a cooling system at $100 \mathrm{~V}$ for $2 \mathrm{~h}$. Membranes were blocked by $5 \%$ non-fat dried milk in Tris-buffered saline with $0.1 \%$ Tween (TBS-T) for $1 \mathrm{~h}$ at room temperature. Membranes were then incubated with anti-GAPDH (diluted 1:20,000), anti-LDLR (diluted 1:200), anti-LXR $\alpha$ (diluted 1:1,000) or anti-Idol (diluted 1:1,000) overnight at $4^{\circ} \mathrm{C}$, washed three times with TBS-T and incubated with peroxidase-conjugated secondary antibodies (anti-mouse or anti-rabbit, respectively, diluted 1:20,000) for $1 \mathrm{~h}$ at room temperature. Specific bands were then detected by the ECL western blot system. Antibodies against GAPDH were used as the normalizing control.

RNA isolation, $c D N A$ synthesis and quantitative polymerase chain reaction $(q P C R)$. Total RNA was isolated with the Ultrapure RNA kit and cDNA synthesized with the RevertAid First Strand cDNA synthesis kit from $1 \mu \mathrm{g}$ total RNA. Primers for mRNA detection were designed and synthesized by Sangon Biotech (Shanghai, China). qPCR was carried out on a CFX96 real-time machine (Bio-Rad) using the SYBR Green polymerase by the $\Delta \Delta \mathrm{Ct}$ method. Values were normalized to GAPDH levels.

Statistical analysis. Duplicates were used in all experiments and experiments were repeated at least three times. Significant differences between the control and treatment groups were assessed by one-way ANOVA with a Bonferroni post hoc test. $\mathrm{P}<0.05$ was considered to indicate a statistically significant difference.

\section{Results}

DHA increases the amount of LDLR protein in a dose-dependent manner. To examine the effect of DHA on the expression of LDLR in vitro, the abundance of LDLR in HepG2 cells was detected by western blot analysis following exposure to the respective fatty acid for $24 \mathrm{~h}$. Compared with HepG2 cells cultured with DMEM, LDLR protein decreased by $77 \%$ $(\mathrm{P}<0.05)$ when treated with 25-hydroxycholesterol $(10 \mu \mathrm{mol} / \mathrm{l}$; Fig. 1A). In the presence of 25-hydroxycholesterol, HepG2 cells were treated with BSA vehicle, PA and DHA, respectively. BSA plus 25-hydroxycholesterol had no significant effect on LDLR protein compared with 25-hydroxycholesterol alone. With co-treatment of 25-hydroxycholesterol, DHA, but not PA, upregulated LDLR protein levels 1.4-fold compared with control cells treated with BSA vehicle (i.e., DHA significantly attenuated the suppressive effects of 25-hydroxycholesterol on LDLR protein abundance; Fig. 1A). However, there was no significant difference in LDLR expression between the cells treated with DHA and with DMEM only (data not shown).

Experiments were performed to determine LDLR expression in response to various concentrations of DHA with 25-hydroxycholesterol co-treatment. At a concentration of $50 \mu \mathrm{mol} / \mathrm{l}$, the amount of LDLR protein did not differ from that observed in control cells treated with BSA vehicle. A dose-dependent increase in LDLR protein abundance was only observed in DHA-treated cells above concentrations of $100 \mu \mathrm{mol} / 1$, confirming its inductive effect on LDLR expression (Fig. 1B).

DHA has no significant effect on the regulation of LDLR $m R N A$. To study whether the increase in LDLR protein levels by DHA was due to the upregulation of LDLR gene expression, the amount of LDLR mRNA was quantified by qPCR. Compared with HepG2 cells cultured with DMEM, LDLR mRNA decreased by $72 \%(\mathrm{P}<0.05)$ when treated with 25-hydroxycholesterol (10 $\mu \mathrm{mol} / 1$; Fig. 2). With co-treatment of 25-hydroxycholesterol, neither DHA nor PA had a significant effect on LDLR mRNA compared with control cells treated with BSA vehicle (Fig. 2). Despite a DHA-induced moderate increase in LDLR protein abundance, DHA did not signifi- 
A
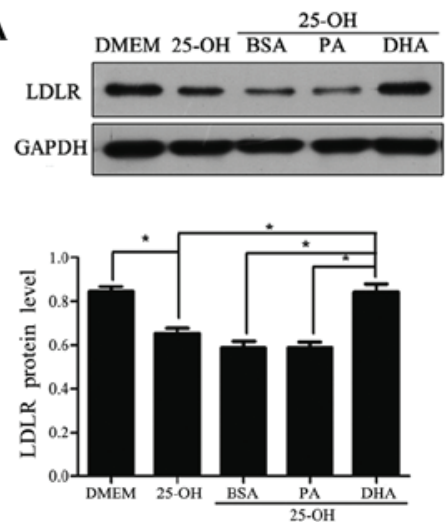

B
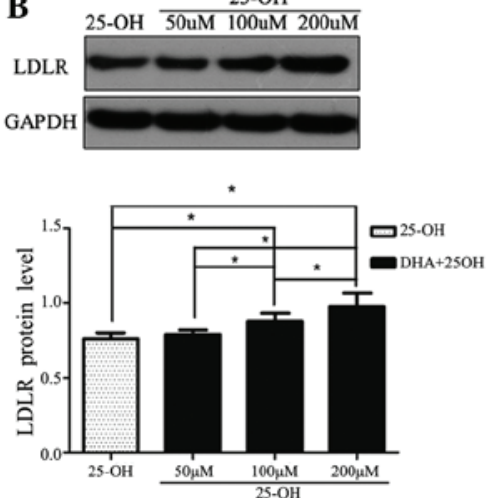

Figure 1. Regulatory effects of LDLR protein on fatty acid treatment. Following overnight culture in a serum-free medium, HepG2 cells were exposed to 25-hydroxycholesterol $(10 \mu \mathrm{mol} / \mathrm{l})$ with or without fatty acid/BSA complexes $(100 \mu \mathrm{mol} / \mathrm{l})$ for $24 \mathrm{~h}$ (A). HepG2 cells were exposed to 25 -hydroxycholesterol $(10 \mu \mathrm{mol} / \mathrm{l})$ with DHA at various concentrations $(50,100$ and $200 \mu \mathrm{mol} / \mathrm{l})(\mathrm{B})$. At the end of the treatment, total cell lysates were isolated for western blot analysis for LDLR and GAPDH. The data shown are the mean $( \pm$ SEM) of at least three separate experiments $(* P<0.05)$. LDLR, low-density lipoprotein receptor; GAPDH, glyceraldehyde 3-phosphate dehydrogenase; DMEM, Dulbecco's modified Eagle's medium; 25-OH, 25-hydroxycholesterol; BSA, bovine serum albumin; PA, palmitic acid; DHA, docosahexanoic acid.

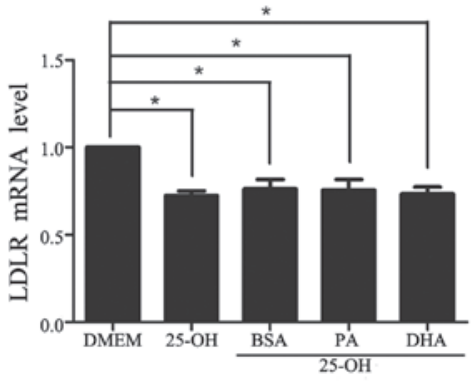

Figure 2. Regulatory effects of LDLR mRNA on fatty acid treatment. Following overnight culture in a serum-free medium, HepG2 cells were exposed to 25 -hydroxycholesterol $(10 \mu \mathrm{mol} / \mathrm{l})$ with or without fatty acid/BSA complexes $(100 \mu \mathrm{mol} / \mathrm{l})$ for $24 \mathrm{~h}$. LDLR mRNA levels were analyzed by quantitative polymerase chain reaction and were normalized to glyceraldehyde 3-phosphate dehydrogenase. The data shown are the mean ( \pm SEM) of at least three separate experiments ("P<0.05). LDLR, low-density lipoprotein receptor; DMEM, Dulbecco's modified Eagle's medium; 25-OH, 25-hydroxycholesterol; BSA, bovine serum albumin; PA, palmitic acid; DHA, docosahexanoic acid.

A
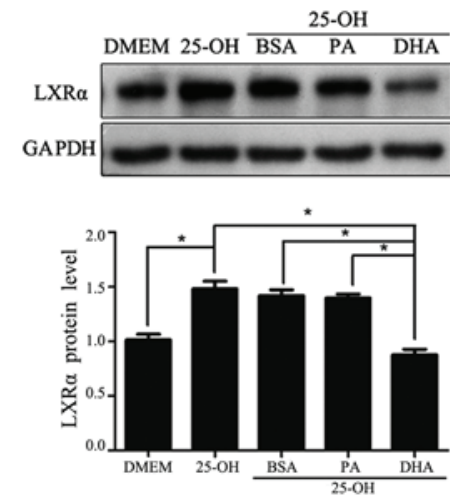

B

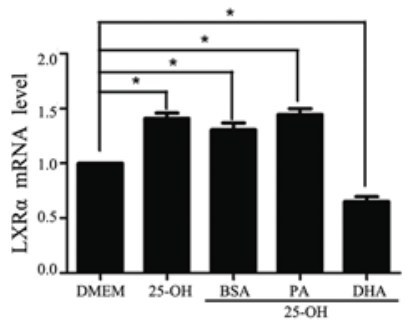

Figure 3. Regulatory effects of LXR $\alpha$ expression on fatty acid treatment. Following overnight culture in a serum-free medium, HepG2 cells were exposed to 25-hydroxycholesterol $(10 \mu \mathrm{mol} / \mathrm{l})$ with or without fatty acid/BSA complexes $(100 \mu \mathrm{mol} / \mathrm{l})$ for $24 \mathrm{~h}$. At the end of the treatment, total cell lysates were isolated for western blot analysis for LXR $\alpha$ and GAPDH (A), and total RNA was isolated for quantitative polymerase chain reaction analysis of target genes (B). The data shown are the mean ( \pm SEM) of three separate experiments ("P<0.05). LXR $\alpha$, liver X receptor $\alpha$; GAPDH, glyceraldehyde 3-phosphate dehydrogenase; DMEM, Dulbecco's modified Eagle's medium; 25-OH, 25-hydroxycholesterol; BSA, bovine serum albumin; PA, palmitic acid; DHA, docosahexanoic acid.

cantly antagonize the inhibition of 25-hydroxycholesterol on LDLR mRNA, even at the highest dose applied.

DHA exerts a downregulatory effect on the expression of $L X R \alpha$. The expression of LXR $\alpha$ was detected on the mRNA and protein levels. Compared with HepG2 cells cultured with DMEM, LXR $\alpha$ protein was increased 1.4-fold $(\mathrm{P}<0.05)$ when treated with 25-hydroxycholesterol (10 $\mu \mathrm{mol} / \mathrm{l}$; Fig. 3A). With co-treatment of 25-hydroxycholesterol, DHA, but not PA, significantly downregulated the protein level of LXR $\alpha$ by $50 \%$ 
A
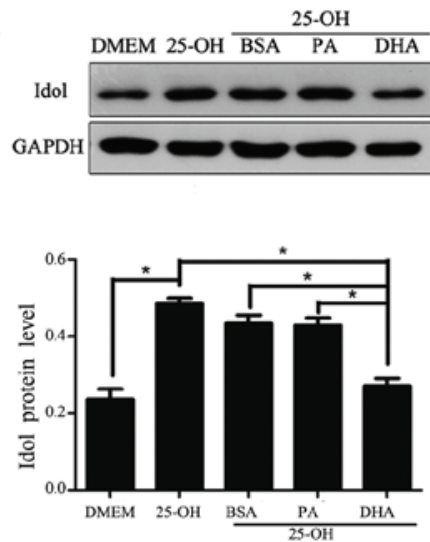

B
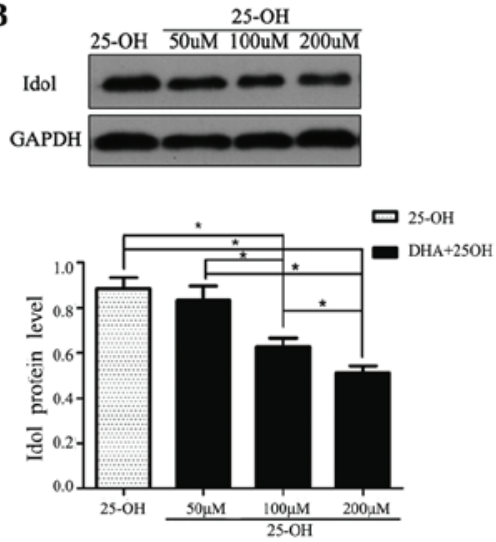

Figure 4. Regulatory effects of Idol protein on fatty acid treatment. Following overnight culture in a serum-free medium, HepG2 cells were exposed to 25-hydroxycholesterol $(10 \mu \mathrm{mol} / \mathrm{l})$ with or without fatty acid/BSA complexes $(100 \mu \mathrm{mol} / \mathrm{l})$ for $24 \mathrm{~h}$ (A). HepG2 cells were exposed to 25 -hydroxycholesterol $(10 \mu \mathrm{mol} / \mathrm{l})$ with DHA at various concentrations (50, 100 and $200 \mu \mathrm{mol} / \mathrm{l})$ (B). At the end of the treatment, total cell lysates were isolated for western blot analysis for Idol and GAPDH. The data shown are the mean ( \pm SEM) of at least three separate experiments ("P $<0.05)$. Idol, inducible degrader of the low-density lipoprotein receptor; GAPDH, glyceraldehyde 3-phosphate dehydrogenase; DMEM, Dulbecco's modified Eagle's medium; 25-OH, 25-hydroxycholesterol; BSA, bovine serum albumin; PA, palmitic acid; DHA, docosahexanoic acid.

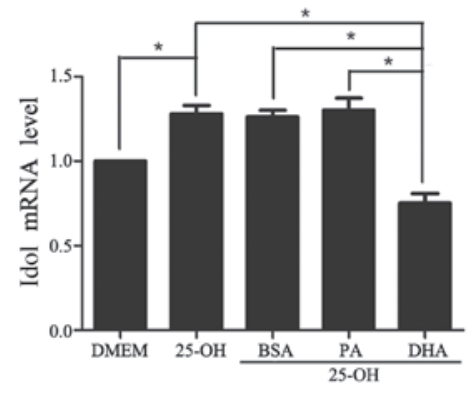

Figure 5. Regulatory effects of Idol mRNA on fatty acid treatment. Following overnight culture overnight in a serum-free medium, HepG2 cells were exposed to 25 -hydroxycholesterol $(10 \mu \mathrm{mol} / \mathrm{l})$ with or without fatty acid/BSA complexes $(100 \mu \mathrm{mol} / \mathrm{l})$ for $24 \mathrm{~h}$. Idol mRNA levels were analyzed by quantitative polymerase chain reaction and were normalized to glyceraldehyde 3 -phosphate dehydrogenase. The data shown are the mean $( \pm$ SEM) of three separate experiments ( $\mathrm{P}<0.05)$. Idol, inducible degrader of the low-density lipoprotein receptor DMEM, Dulbecco's modified Eagle's medium; 25-OH, 25-hydroxycholesterol; BSA, bovine serum albumin; PA, palmitic acid; DHA, docosahexanoic acid.

compared with control cells treated with BSA vehicle. Parallel alteration was observed with the LXR $\alpha$ mRNA levels (Fig. 3B).

DHA decreases the expression of Idol in a dose-dependent manner. Since DHA exerted a downregulatory effect on the expression of $\operatorname{LXR} \alpha$, we evaluated the change in Idol, which is the downstream protein of the nuclear receptor $\mathrm{LXR} \alpha$. As expected, the expression of Idol was increased by 25-hydroxycholesterol and decreased by DHA on the mRNA and protein levels, by $60 \%$ and $62 \%$, respectively, in coordination with the change in LXR $\alpha$ expression (Figs. 4 and 5). In addition, a dose-dependent decrease in the amount of Idol protein was observed with the various doses of DHA (Fig. 4B).

\section{Discussion}

Idol has been identified as a novel post-transcriptional regulator of LDLR abundance, as its full name implies. Containing a unique C-terminal RING domain, Idol is an E3 ubiquitin ligase that triggers ubiquitination of LDLR and promotes its internalization and degradation (11,17). Distinct from LDLR and PCSK9 genes, Idol is directly regulated by LXR $\alpha$, which is activated in response to cellular cholesterol excess (12). Conversely, the expression of SREBP2 is responsive to cellular cholesterol depletion (7). Therefore, the LXR $\alpha$-Idol-LDLR pathway and the SREBP2-PCSK9-LDLR pathway are complementary but independent pathways in the response to cellular sterol status.

As one type of oxysterol, 25-hydroxycholesterol strongly represses the SREBP2 process and slightly activates the LXR $\alpha$ pathway (18). Since LDLR is the downstream protein in the two pathways, the net effect of 25-hydroxycholesterol is the downregulation of LDLR abundance. Therefore, in the present study, HepG2 cells were initially treated with 25-hydroxycholesterol to decrease basal levels of the LDLR protein as previously described (16). In the presence of 25-hydroxycholesterol, DHA significantly increased LDLR protein level in a dose-dependent manner over $100 \mu \mathrm{mol} / \mathrm{l}$.

Although numerous in vivo and in vitro studies have been conducted, the mechanism by which omega-3 fatty acids exerted their effect on LDLR expression remained unclear. Previous studies have indicated that multiple mechanisms are involved in regulating the LDLR gene independently of SREBP1 $(16,19)$. Conversely, a number of studies demonstrated that DHA inhibited lipogenic gene transcription by suppressing the expression of SREBP1, possibly at the post-transcriptional level $(20,21)$. There was no evidence that LDLR played a role in this; however, DHA downregulated the hepatic mRNA of SREBP2, and LDLR was observed in hamsters fed a high cholesterol diet (22).

The present study revealed that DHA had no effect on LDLR mRNA levels even at the highest dose applied, suggesting that DHA may affect LDLR via mechanisms other than gene expression. In addition, a number of studies demonstrated that DHA inhibits the activity of $\operatorname{LXR} \alpha(13,14)$. Furthermore, LXR $\alpha$ controls the activation of the transcription of Idol (11). Therefore, we speculated that the unclear mechanism by which DHA increased LDLR abundance is most likely mediated by suppression of the LXR $\alpha$-Idol pathway. 
When delivered with the appropriate treatment, we observed that 25-hydroxycholesterol activated LXR $\alpha$ expression since it is an agonist of LXR $\alpha$. In line earlier findings, DHA significantly repressed the expression of LXR $\alpha$ at the mRNA and protein levels when administered with 25-hydroxycholesterol (16). As expected, the LXR $\alpha$ target gene Idol was significantly decreased on DHA treatment. Consistent with the change in mRNA levels, DHA reduced Idol protein. Moreover, the reduction of Idol abundance presented in a dose-dependent manner, corresponding with the alteration of LDLR protein but in the opposite manner.

All of these results confirmed that DHA suppressed the LXR $\alpha$-Idol pathway, and in turn lowered the Idol-induced degradation of LDLR protein, leading to the upregulation of LDLR.

However, there was no significant difference in the LDLR abundance between DHA treatment and BSA vehicle or PA treatment when 25-hydroxycholesterol was absent. We therefore speculated that DHA possibly exerted an upregulatory effect of LDLR abundance under the condition of high cholesterol levels. In our study, DHA significantly attenuated the suppression effect of 25-hydroxycholesterol on LDLR abundance, as well as the LXR $\alpha$-Idol pathway. That is, DHA modified LDLR abundance via suppression of the LXR $\alpha$-Idol pathway. The findings of the present study suggest that in addition to the suppression of the LXR $\alpha$-Idol pathway, there are multiple mechanisms participating in the regulation of LDLR by DHA treatment, and further exploration is required.

In conclusion, we identified that DHA increased hepatic LDLR abundance in the presence of 25-hydroxycholesterol. Multiple mechanisms are involved in DHA regulating the LDLR abundance, and the suppression of LXR $\alpha$-Idol pathway is one such mechanism.

\section{References}

1. Stone NJ, Robinson JG, Lichtenstein AH, et al: 2013 ACC/AHA guideline on the treatment of blood cholesterol to reduce atherosclerotic cardiovascular risk in adults: a report of the American College of Cardiology/American Heart Association Task Force on Practice Guidelines. J Am Coll Cardiol 63: 2889-2934, 2014.

2. Amarenco P, Labreuche J, Lavallée P and Touboul PJ: Statins in stroke prevention and carotid atherosclerosis: systematic review and up-to-date meta-analysis. Stroke 35: 2902-2909, 2004.

3. ACCORD Study Group; Ginsberg HN, Elam MB, Lovato LC, et al: Effects of combination lipid therapy in type 2 diabetes mellitus. N Engl J Med 362: 1563-1574, 2010.

4. Ingelsson E, Schaefer EJ, Contois JH, et al: Clinical utility of different lipid measures for prediction of coronary heart disease in men and women. JAMA 298: 776-785, 2007.
5. Pedersen TR, Faergeman O, Kastelein JJ, et al: High-dose atorvastatin vs usual-dose simvastatin for secondary prevention after myocardial infarction: the IDEAL study: a randomized controlled trial. JAMA 294: 2437-2445, 2005.

6. Yamamoto T, Davis CG, Brown MS, et al: The human LDL receptor: a cysteine-rich protein with multiple Alu sequences in its mRNA. Cell 39: 27-38, 1984.

7. Hua X, Yokoyama C, Wu J et al: SREBP-2, a second basic-helix-loop-helix-leucine zipper protein that stimulates transcription by binding to a sterol regulatory element. Proc Natl Acad Sci USA 90: 11603-11607, 1993.

8. Kawabe Y, Suzuki T, Hayashi M, Hamakubo T, Sato R and Kodama T: The physiological role of sterol regulatory element-binding protein-2 in cultured human cells. Biochim Biophys Acta 1436: 307-318, 1999.

9. Jeong HJ, Lee HS, Kim KS, Kim YK, Yoon D and Park SW: Sterol-dependent regulation of proprotein convertase subtilisin/kexin type 9 expression by sterol-regulatory element binding protein-2. J Lipid Res 49: 399-409, 2008.

10. Fisher TS, Lo Surdo P, Pandit S, et al: Effects of $\mathrm{pH}$ and low density lipoprotein (LDL) on PCSK9-dependent LDL receptor regulation. J Biol Chem 282: 20502-20512, 2007.

11. Zelcer N, Hong C, Boyadjian R and Tontonoz P: LXR regulates cholesterol uptake through Idol-dependent ubiquitination of the LDL receptor. Science 325: 100-104, 2009.

12. Zelcer $\mathrm{N}$ and Tontonoz P: Liver $\mathrm{X}$ receptors as integrators of metabolic and inflammatory signaling. J Clin Invest 116: 607-614, 2006.

13. Pawar A, Botolin D, Mangelsdorf DJ and Jump DB: The role of liver $\mathrm{X}$ receptor-alpha in the fatty acid regulation of hepatic gene expression. J Biol Chem 278: 40736-40743, 2003.

14. Vanden Heuvel JP: Cardiovascular disease-related genes and regulation by diet. Curr Atheroscler Rep 11: 448-455, 2009.

15. Mustad VA, Ellsworth JL, Cooper AD, Kris-Etherton PM and Etherton TD: Dietary linoleic acid increases and palmitic acid decreases hepatic LDL receptor protein and mRNA abundance in young pigs. J Lipid Res 37: 2310-2323, 1996.

16. Yu-Poth S, Yin D, Kris-Etherton PM, Zhao G and Etherton TD: Long-chain polyunsaturated fatty acids upregulate LDL receptor protein expression in fibroblasts and HepG2 cells. J Nutr 135: 2541-2545, 2005.

17. Sorrentino V, Scheer L, Santos A, Reits E, Bleijlevens B and Zelcer N: Distinct functional domains contribute to degradation of the low density lipoprotein receptor (LDLR) by the E3 ubiquitin ligase inducible Degrader of the LDLR (IDOL). J Biol Chem 286: 30190-30199, 2011.

18. Janowski BA, Willy PJ, Devi TR, Falck JR and Mangelsdorf DJ: An oxysterol signalling pathway mediated by the nuclear receptor LXR alpha. Nature 383: 728-731, 1996.

19. Field FJ, Born E and Mathur SN: Fatty acid flux suppresses fatty acid synthesis in hamster intestine independently of SREBP-1 expression. J Lipid Res 44: 1199-1208, 2003.

20. Xu J, Nakamura MT, Cho HP and Clarke SD: Sterol regulatory element binding protein-1 expression is suppressed by dietary polyunsaturated fatty acids. A mechanism for the coordinate suppression of lipogenic genes by polyunsaturated fats. J Biol Chem 274: 23577-23583, 1999.

21. Xu J, Teran-Garcia M, Park JH, Nakamura MT and Clarke SD: Polyunsaturated fatty acids suppress hepatic sterol regulatory element-binding protein-1 expression by accelerating transcript decay. J Biol Chem 276: 9800-9807, 2001.

22. Chen J, Jiang Y, Liang Y, et al: DPA n-3, DPA n-6 and DHA improve lipoprotein profiles and aortic function in hamsters fed a high cholesterol diet. Atherosclerosis 221: 397-404, 2012. 